\title{
Experimental Study on the Improvement of Schisandra and Epimedium Extract on the Memory Ability in Mice
} Jiahong $\mathrm{Li}^{1,2, \mathrm{a}}$, Chunmei Wang ${ }^{1, \mathrm{~b} \triangle}$,Bing $\mathrm{Li}^{3, \mathrm{c}}$, Jinghui Sun ${ }^{1, \mathrm{~d}}$, Weijing Sun ${ }^{1, \mathrm{e}}$,

\author{
$\mathrm{He} \mathrm{Li}^{1, \mathrm{f} .}$ and Jianguang Chen ${ }^{1, \mathrm{~g} .}$ \\ ${ }^{1}$ College of Pharmacy, Beihua University, Jilin City, 132013, China \\ ${ }^{2}$ Jilin engineering vocational college,Siping City,136000, China \\ ${ }^{3}$ Basic Medical College, Beihua University, Jilin City, 132013, China

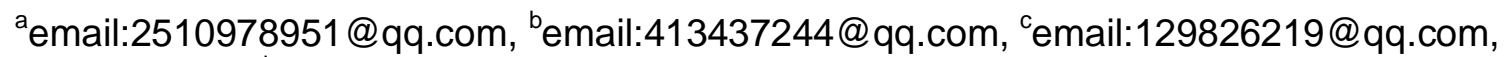 \\ demail:893716972@qq.com, eemail:2316346720@qq.com, \\ f email:yitonglh@126.com, corresponding author, \\ gemail: 644257703@qq.com, corresponding author,
}

co-first author, corresponding author

\begin{abstract}
Keywords: Schisandra; Epimedium; Memory; MDA; SOD.
Abstract. To observe the improvement of Schisandra and Epimedium extract (WY) on the memory ability in mice. Methods: ICR male mice were randomly divided into control group, low-, mediumand high-dose WY groups. The mice were intragastrically given the same volume of distilled water, $300 \mathrm{mg} / \mathrm{kg} \mathrm{WY}, 600 \mathrm{mg} / \mathrm{kg} \mathrm{WY}$, and $1200 \mathrm{mg} / \mathrm{kg}$ WY one time daily for 28 days, respectively. On the first day after the administration, the step-down test were performed to observe the learning and memory ability of mice; moreover, SOD activities and MDA contents in brain tissues of the mice were further detected to explore the underlying mechanisms of effects of WY on the mice's memory ability. Results: Compared with those in the blank control group, the memory ability of mice in WY-treated groups were significantly improved, and the content of MDA was decreased and SOD activity was increased in WY-treated groups $(\mathrm{p}<0.05)$. Conclusion: WY can improve the mice's memory ability .
\end{abstract}

\section{Introduction}

Human memory disorder is an important medical and psychological problem. The development of safe and effective Chinese medicine health foods with the improvement of learning and memory function based on the theory of traditional Chinese medicine and using the modern medical technology has shown a profound social value, and a good economic benefit. in the genus Schisandra (Schisandra chinensis Turcz Baill), a Magnoliaceae plant, is a genuine traditional Chinese medicine in Jilin area, with antioxidant, anti-aging, anti fatigue, immune enhancement, and other biological activities [1-3]; Epimedium (Aceranthus sagittatus S. et Z) is a Berberidaceae Epimedium plant, with anti-aging and immune enhancement [4-7]. In this study, effects of mixed Schisandra and Epimedium extract (WY) on the memory ability were investigated in mice, in order to provide the experimental evidence for the research and development of a health food with an auxiliary improvement on the memory ability.

\section{Materials and Methods}

\section{experimental animals}

ICR Mice, weighing $20 \pm 2 \mathrm{~g}$, provided by Changchun Yisi Experimental Animal Research Center, were raised at $20-25{ }^{\circ} \mathrm{C}$ in separate cages, eating and drinking freely, and for 5 days to acclimate the environment.

\section{Drugs, Reagents and Instruments}


The Schisandra and Epimedium extract (WY) is a mixture of Chinese herbalmedicines Schisandra and Epimedium extracts, provide by The Schisandra development and Industralization Research Center of Jilin Province. Mouse MDA and SOD activity assay kits were purchased from Shanghai Biyuntian Biotechnology Co. ltd.. BA-200 and DT-200 mice step-through instruments were purchased from Chengdu Taimeng Technology Co., ltd..

\section{Grouping of experimental animals and administration}

ICR male mice were randomly divided into 4 groups: blank control group,low-dose WY (300 $\mathrm{mg} / \mathrm{kg})$, medium-dose WY (600 mg/kg), and high-dose WY (1200 mg/kg). Mice in each WY-treated group were given the corresponding doses of WY in a volume of $10 \mathrm{~mL} / \mathrm{kg}$ intragastrically, and those in the blank control group was given the equal volume of distilled water in the same way, 1 time daily, for 28 days. Learning and memory abilities, MDA contents and SOD activities in brain of the mice were measured one hour after the administration.

\section{Step-down test in mice}

In the test, the mice were placed on the copper grid and subjected to electric shocks when the copper grid was charged with electricity; the normal reaction of mice when they were hurt by the electric shock was to jump on the platform to avoid electric shock; most of them often jumped off the platform one more time or repeatedly to be hurt by the electric shock and then quickly jumped back to the platform. The mice were trained in this way for $5 \mathrm{~min}$. $24 \mathrm{~h}$ Later, the test was repeated. In the repeated test, the mice were first placed on the platform, and then, the time of the first jumping off the platform (latency) of mice and the number of errors within $3 \mathrm{~min}$ were recorded, which were considered to reflect mice's memory retention.

\section{Determination of SOD and MDA contents in the brain tissues}

The mice were sacrificed with cervical dislocation, and then the brain tissues were quickly removed and weighed. SOD activities and MDA content in the brain tissues of mice were detected according to instructions of the kits.

\section{Statistical analysis}

SPSS 13.0 software was applied for the statistical analysis. All the data were described as mean \pm SE. The data among multiple groups were compared with One Way ANOVA, and $\mathrm{P}<0.05$ meant a significant difference.

\section{Results}

\section{Step-down test in mice}

As shown in Fig.1, on the second day of the memory test, compared with those in the control group, Latency were significantly prolonged $(\mathrm{P}<0.05)$ after WY were administered, and numbers of error within 5 min were significantly reduced $(\mathrm{P}<0.05)$ in mice in medium-dose WY group. The results indicate that WY can improve memory abilities of mice.
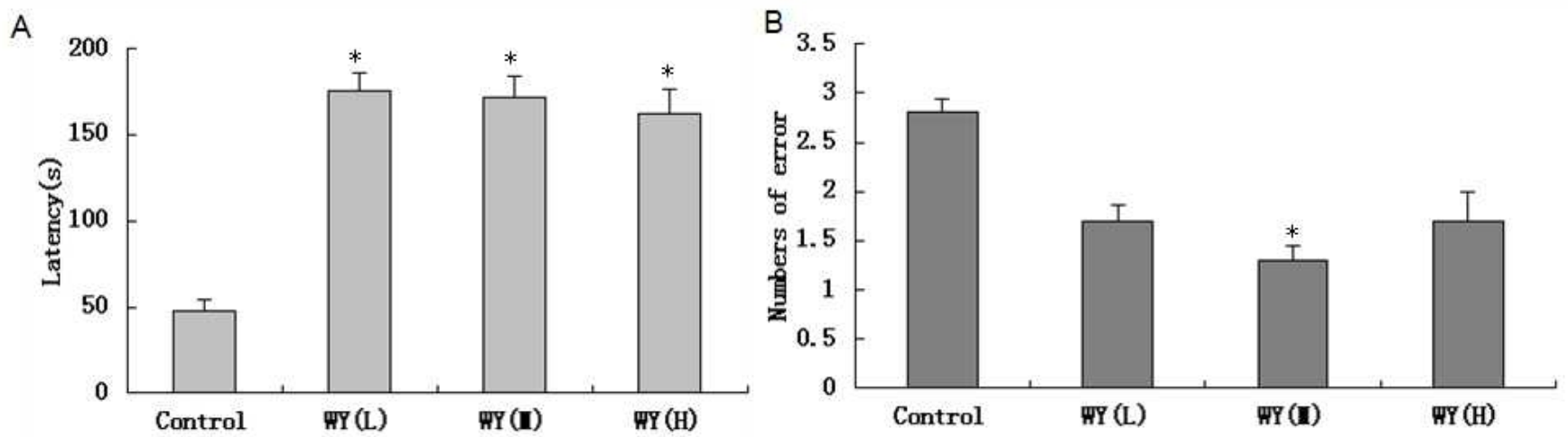

Fig.1 Effect of WY on memory ability in mice

Note: A. Latency; B. Numbers or error . ${ }^{*}$ Compared with those in the control group, $\mathrm{P}<0.05$. 


\section{Effects of WY on MDA contents and SOD activities in the mice's brain tissues}

As shown in Fig.2, compared with those in the blank control group, MDA contents in the brain tissues of mice in low-dose WY group were not changed obviously, but those in the brain tissues in medium-dose and high-dose groups were lowered significantly $(\mathrm{P}<0.05)$; compared with those in the blank control group, SOD activites in brain tissues of the mice in medium- and high-dose groups were elevated.
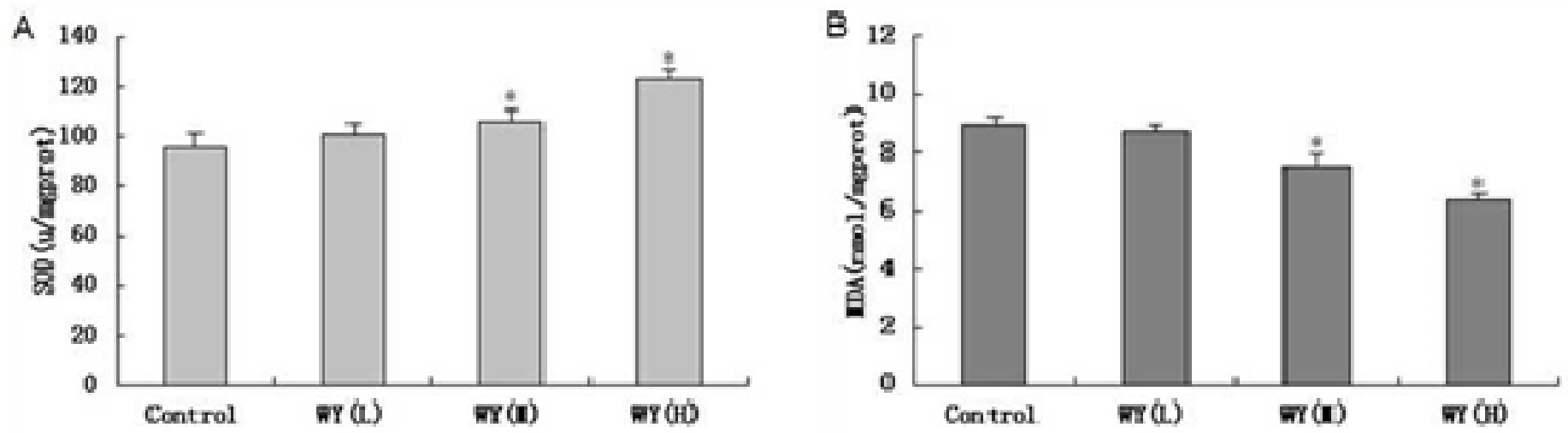

Fig.2 Effects of WY on MDA contents and SOD activities in the mice's brain tissues

Note: A. SOD activity; B. MDA cotents . ${ }^{*}$ Compared with those in the control group, $\mathrm{P}<0.05$.

\section{Discussion}

In this study, a step-down test and a passive avoidance test were performed in mice, and MDA contents and SOD activities in the mice's brain tissues were detected for the comprehensive assessment of effects of WY on the memory ability in mice. The results indicate that WY has an auxiliary improvement on the memory function in mice. The Schisandra and Epimedium extract (WY) used in this study was an alcohol extract of Schisandra and Epimedium compound, and its specific formula and extraction method have been published in other papers.

In the mice step-down test, mice are placed on a power grid with a certain voltage, and when an electrical stimulation with a certain intensity is given, the mice will search for a safe region in order to avoid the hurt of electricity; the mice can finally remember the safe region after they experience this several times repeatedly, which can thus reflect the process of mice's memory acquisition, consolidation and reproduction. In this study, the memory ability of mice in the different groups was assessed by the step-down test. The results showed that the memory ability of mice in WY-treated groups were significantly improved compared with those in the blank control group, indicating that WY could improve the memory ability of mice.

SOD (superoxide dismutase) is an important antioxidase in living body, which can scavenge superoxide anion free radicals through its dismutation, so as to play its function of protecting the body's cells. The experimental results showed that WY could increase SOD activities and decrease MDA contents in brain tissues of the mice, suggesting that WY may improve the mice's memory ability by reducing the peroxidation of lipids in the mice's brain. However, this hypothesis needs to be confirmed by further experiments.

\section{Acknowledgements}

This research work was supported by the projects of "Science and Technology Department of Jilin Province".

$\mathrm{He} \mathrm{Li}$ and Jianguang Chen are both the corresponding authors of this article. 


\section{References}

[1] W.J. Sun, W.W. Lu, X.F. Yu, Z.Z. Lu, S. Wang, The experimental study of CPSC on anti-aging. Chin. J. Gerontol. 21 (2001) 454-455.

[2] G.Q. Ying, Z.M. Yu, J.F. Shan, Y. Yi, Research progresses of the effective components of Schisandra chinensis. Henan. Med. Res. 24 (2005) 84-87.

[3] B.L. Guo, P.G. Xiao, Comment on main species of Herba Epimedii. Chin. J. Chin. Mater. Med. 28 (2003) 303-307.

[4] L.J. Wang, Q.Y. Wang, Y. Wang, R. Li, M.B. Xie, Effect of Herba Epimedii on learning and memory ability of mice with memory disorders. Chin. J. Basic. Med. Tradit. Chin. Med. 18 (2012) 665-674.

[5] L Hritcu , M Stefan , R Brandsch , M Mihasan. Enhanced behavioral response by decreasing brain oxidative stress to 6-hydroxy-1-nicotine in Alzheimer's disease rat model. J. Neurosci. Lett. 591(2015) 41-47.

[6] H.F. Wang, C. Xu, M.L. Zhou, et al. Studies on Improving the Memory Capacity of Mice. J. J. Chin. Cereal Oil. Assoc.27(2012) 63-66.

[7] W.L. Shi, Z.Y. Yan, C.F. Cao, F. Wang, Z.J. Yang. Improvement of Pine Pollen on Learning and Memory Disorder of Mice Caused by Lead Poisoning. J. Food Sci. 35(2014)277-279. 